\title{
Liquid fuel ignition features during its spilling on the metallic substrate heated up to high temperatures
}

\author{
Olga Vysokomornaya, Anastasia Scherbinina and Pavel Strizhak ${ }^{\mathrm{a}}$ \\ National Research Tomsk Polytechnic University, 634050 Tomsk, Russia
}

\begin{abstract}
Forecasting heat and mass transfer model for the numerical investigation of liquid fuel ignition features during its spilling on the metallic substrate heated up to high temperatures was developed. The dependences of liquid fuel (by the example of kerosene) ignition delay times on the velocity of its spilling, the liquid film (which is formed) thickness and the metallic substrate temperature were found. The least values of these parameters (whereby the ignition is possible under the heat and mass transfer conditions considered) were determined.
\end{abstract}

\section{Introduction}

The analysis of historical data shows that the number of fires and explosions at the places of production associated with the usage of flammable and combustible liquid matters is steadily increased over the last years. The broad-scale research carrying out of macroscopic regularities of ignition and combustion of liquids in its coordination with different, primarily, typical heating sources with the purpose of the determination of the safe conditions for production, storage, transportation, transshipment and allocation of liquid matters is necessary under such conditions (not to mention the requirement strengthening on the part of fire protection agency, routine and discipline strengthening at the places of production). It should be pointed out that the uncontrollable processes of liquid fuel pouring and the matters unappropriated for combustion represent the maximal fire and explosion hazard, as a rule. According to such conditions the ignition can occur in coordination with a heated body, spark, exposed conductors, direct flame and other energy sources. Probably, the interacting processes of spilling liquids with the working areas (heated up to high temperatures) of equipment and metal constructions are the most typical ignition conditions. The "liquid film - substrate" systems are used as often as not in the numerical analysis of such processes. At that, the emphasis, as a rule, is on the stationary-state combustion. The analysis of ignition conditions for the spilling liquid fuel on the surface heated up to high temperatures is of interest.

The purpose of the article is the numerical investigation of macroscopic regularities of typical liquid fuel ignition under its spilling on the metal substrate heated up to high temperatures.

\footnotetext{
${ }^{a}$ Corresponding author: pavelspa@tpu.ru
}

This is an Open Access article distributed under the terms of the Creative Commons Attribution License 4.0, which permits unrestricted use, distribution, and reproduction in any medium, provided the original work is properly cited. 


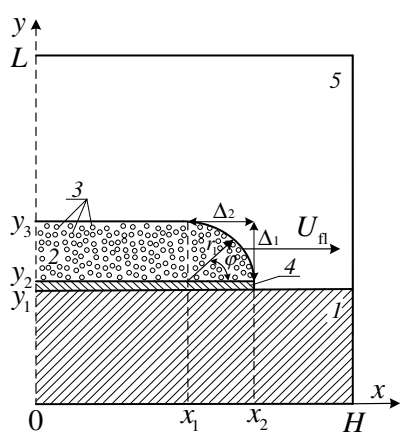

Figure 1. The schematic illustration of the problem solution region at $0<t<t_{\mathrm{d}}$ : 1 - metal substrate, $2-$ liquid fuel, 3 - bubbles of liquid vapors, 4 - vapor gap, 5 - oxidizing agent-liquid vapor mixture.

\section{Problem statement}

The following process scheme being investigated was accepted. A thin layer (thickness $L_{\mathrm{fl}}$ ) of the typical liquid fuel spreads over the metal substrate heated up to high temperature $T_{\mathrm{bm}}$ at a constant speed $U_{\mathrm{fl}}$ (Fig. 1). The initial temperature of liquid fuel layer $T_{0}$ was taken to be equal to the room temperature. The evaporation takes place when achieving of phase transformation conditions, and the vapor gap between spilling liquid layer and the substrate is formed. The wall layer of liquid fuel is warmed up to the boiling temperature $T_{\mathrm{bl}}$. Consequently, the bubbles with liquid vapors from the vapor gap area (Fig. 1) soar by the action of buoyancy force, get through the matter layer and are blown into gas area. The liquid vapors are mixed with the oxidizing agent by diffusion and convection. The vapor-gas mixture is formed. The oxidation reaction takes nonreversible drastically exponential form when achieving the limit concentration values of the constituents and the vapor-gas mixture temperature (the ignition takes place).

The following allowances unapplied significant restrictions to the problem statement were accepted during the numerical simulation:

1. The thermal and physical characteristics of reacting agents do not depend on temperature. It is found [1-3] that the dependences of thermal and physical parameters on temperature influence slightly on the integral characteristics of evaporation and ignition processes of typical liquid fuels.

2. The one matter with the known characteristics is formed in consequence of the combustible liquid evaporation. The "effective" values of activation energy $E$ and preexponential factor $k_{0}$ are generally defined when determining (experimentally) of kinetic oxidation reaction parameters of liquid matter vapors. Therefore, the implementation of one "effective" reaction, wherein one combustible liquid takes part, is expected.

3. The liquid boiling and spilling do not lead to the fuel layer thickness variation (loss in liquid fuel weight is balanced out by its inflow at a constant speed $U_{\mathrm{fl}}$ ).

4. The possible liquid burning-out processes were not taken into account. It was thought that the liquid fuel film poured on the substrate surface occupies significantly large area.

5. The front of spilling liquid layer (Fig. 1) has the shape of a spherical segment (radius $r_{1}$ is equal to the liquid layer thickness). The analysis of liquid droplet spilling conditions on the metal substrates [4] carries inference that the hemispheric model can be used as a first approximation in the system under consideration (Fig. 1). 
The following ignition conditions were accepted during the numerical simulation [5]:

1. The heat released in consequence of the chemical oxidation reaction of fuel vapors in the air is more than the heat transmitted from the substrate to the boiling liquid and the vapor-gas mixture.

2. The mixture temperature of fuel vapors and air exceeds the temperature of massive substrate.

Theoretical investigations of interconnected heat and mass transfer process in the system (Fig. 1) were carried out for parallelepiped-shape particle with sizes $L_{\mathrm{p}}\left(L_{\mathrm{p}}=X_{1}\right)$ and $H_{\mathrm{p}}\left(H_{\mathrm{p}}=Y_{4}-Y_{2}\right)$. Sizes of solution area $L$ and $H$ were changed greatly more than $L_{\mathrm{p}}$ and $H_{\mathrm{p}}$.

Follow ignition conditions were assumed [1-3]:

1. Heat released due to chemical reaction of fuel vapors oxidation is more than heat transferred from a hot particle to liquid condensed substance and air.

2. Temperature of gas-vapor mixture exceeds the initial temperature of a particle.

\section{Mathematical model and solution method}

The mathematical model corresponding to the accepted problem statement (Fig. 1) includes the following system of nonlinear nonstationary partial differential equations $\left(0<t<t_{\mathrm{d}}\right)$ written in accordance with the conceptual issues of diffusion and heat transfer theories in the chemical kinetics:

$0<x<H, 0<y<y_{1}$

$$
C_{1} \rho_{1} \frac{\partial T_{1}}{\partial t}=\lambda_{1}\left[\frac{\partial^{2} T_{1}}{\partial x^{2}}+\frac{\partial^{2} T_{1}}{\partial y^{2}}\right]
$$

$0<x<x_{1}, y_{2}<y<y_{3}$

$$
\begin{gathered}
C_{2} \rho_{2} \frac{\partial T_{2}}{\partial t}=\lambda_{2}\left[\frac{\partial^{2} T_{2}}{\partial x^{2}}+\frac{\partial^{2} T_{2}}{\partial y^{2}}\right] \text { at } T<T_{\mathrm{bl}}, \\
C_{2} \rho_{2} \frac{\partial T_{2}}{\partial t}=\lambda_{2}\left[\frac{\partial^{2} T_{2}}{\partial x^{2}}+\frac{\partial^{2} T_{2}}{\partial y^{2}}\right]+C_{4} \rho_{4} V_{\mathrm{vm}} \frac{\partial T_{2}}{\partial y} \text { at } T>T_{\mathrm{bl}} ;
\end{gathered}
$$

$0<r<r_{1}, 0<\varphi<\pi / 2$

$$
\begin{gathered}
\frac{\partial T_{2}}{\partial t}=a_{2}\left[\frac{1}{r^{2}} \frac{\partial}{\partial r}\left(r^{2} \frac{\partial T_{2}}{\partial r}\right)+\frac{1}{r^{2} \sin (\varphi)} \frac{\partial}{\partial \varphi}\left(\sin (\varphi) \frac{\partial T_{2}}{\partial \varphi}\right)\right] \text { at } T<T_{\mathrm{bl}}, \\
\frac{\partial T_{2}}{\partial t}=a_{2}\left[\frac{1}{r^{2}} \frac{\partial}{\partial r}\left(r^{2} \frac{\partial T_{2}}{\partial r}\right)+\frac{1}{r^{2} \sin (\varphi)} \frac{\partial}{\partial \varphi}\left(\sin (\varphi) \frac{\partial T_{2}}{\partial \varphi}\right)\right]+\frac{C_{4} \rho_{4} V_{\mathrm{vm}}}{C_{2} \rho_{2}} \frac{\partial T_{2}}{\partial r} \text { at } T>T_{\mathrm{bl}} ;
\end{gathered}
$$

$0<x<H, y_{1}<y<y_{2} ; x_{1}+\Delta_{2}<x<x_{2}, y_{2}+\Delta_{1}<y<y_{3} ; x_{2}<x<H, y_{2}<y<y_{3} ; 0<x<$ $H, y_{3}<y<L$

$$
\begin{gathered}
\frac{\partial^{2} \psi}{\partial x^{2}}+\frac{\partial^{2} \psi}{\partial y^{2}}=\omega, \\
\frac{\partial \omega}{\partial t}+u \frac{\partial \omega}{\partial x}+w \frac{\partial \omega}{\partial y}=v_{5}\left[\frac{\partial^{2} \omega}{\partial x^{2}}+\frac{\partial^{2} \omega}{\partial y^{2}}\right]+\beta g_{y} \frac{\partial T_{5}}{\partial y}, \\
\rho_{5}\left[\frac{\partial C_{\mathrm{f}}}{\partial t}+u \frac{\partial C_{\mathrm{f}}}{\partial x}+w \frac{\partial C_{\mathrm{f}}}{\partial y}\right]=\rho_{5} D_{5}\left[\frac{\partial^{2} C_{\mathrm{f}}}{\partial x^{2}}+\frac{\partial^{2} C_{\mathrm{f}}}{\partial y^{2}}\right]-W_{\mathrm{o}}, \\
C_{\mathrm{f}}+C_{\mathrm{o}}=1,
\end{gathered}
$$




$$
\rho_{5} C_{5}\left[\frac{\partial T_{5}}{\partial t}+u \frac{\partial T_{5}}{\partial x}+w \frac{\partial T_{5}}{\partial y}\right]=\lambda_{5}\left[\frac{\partial^{2} T_{5}}{\partial x^{2}}+\frac{\partial^{2} T_{5}}{\partial y^{2}}\right]+Q_{\mathrm{o}} W_{\mathrm{o}} .
$$

Here $t$ - time, $\mathrm{s} ; t_{\mathrm{d}}$ - ignition delay time, $\mathrm{s} ; x, y$-Cartesian coordinates; $H, L$ - solution region dimensions, $\mathrm{m} ; C$ - specific heat capacity, $\mathrm{J} /(\mathrm{kg} \cdot \mathrm{K}) ; \rho-$ density, $\mathrm{kg} / \mathrm{m}^{3} ; \lambda$ - thermal conductivity coefficient, $\mathrm{W} /(\mathrm{m} \cdot \mathrm{K}) ; T$ - temperature, $\mathrm{K} ; V_{\mathrm{vm}}$ - vapor bubble-rise velocity, $\mathrm{m} / \mathrm{s} ; r, \varphi$ - spherical system coordinates; $a$ - temperature conductivity coefficient, $\mathrm{m}^{2} / \mathrm{s} ; \Delta_{1}, \Delta_{2}-\operatorname{complexes}\left(\Delta_{1}=r_{1} \sin (\varphi)\right.$, $\left.\Delta_{2}=r_{1} \cos (\varphi)\right), \mathrm{m} ; \psi$ - flow function, $\mathrm{m}^{2} / \mathrm{s} ; \omega$ - vortex velocity vector, $\mathrm{s}^{-1} ; u, w$ - convection velocity components of fuel vapors-oxidizing agent mixture in projections on $x$ and $y$ axes, correspondingly, $\mathrm{m} / \mathrm{s}$; $v$ - kinematic viscosity, $\mathrm{m}^{2} / \mathrm{s} ; \beta$ - thermal-expansion coefficient, $\mathrm{K}^{-1} ; g_{y}$ - gravitational acceleration in projection on $y$ axis, $\mathrm{m} / \mathrm{s}^{2} ; C_{\mathrm{f}}$ - liquid fuel vapor concentration; $D$ - diffusion coefficient of fuel vapors in the air, $\mathrm{m}^{2} / \mathrm{s} ; W_{\mathrm{o}}$ - mass oxidation velocity of fuel vapors in the air, $\mathrm{kg} /\left(\mathrm{m}^{3} \cdot \mathrm{s}\right) ; C_{\mathrm{o}}$ - concentration of oxidizing agent; $Q_{\mathrm{o}}$ - heat effect of oxidation reaction of fuel vapors in the air, $\mathrm{MJ} / \mathrm{kg} ; 1,2,3,4,5$ indexes correspond metal substrate, liquid fuel, oxidizing agent, fuel vapors, vapor-gas mixture (Fig. 1).

The initial conditions (Fig. 1): $T=T_{\mathrm{bm}}$ at $0<x<H, 0<y<y_{1} ; T=T_{0}$ at $0<x<x_{1}, y_{2}<$ $y<y_{3}, 0<r<r_{1}, 0<\varphi<\pi / 2 ; \omega=0, \psi=0, C_{\mathrm{f}}=0, T=T_{0}$ at $0<x<H, y_{1}<y<y_{2} ; x_{1}+\Delta_{2}<$ $x<x_{2}, y_{2}+\Delta_{1}<y<y_{3} ; x_{2}<x<H, y_{2}<y<y_{3} ; 0<x<H, y_{3}<y<L$.

The boundary conditions (Fig. 1): the conjugating boundary conditions were prescribed on "substrate - liquid fuel vapors" boundary $\left(y=y_{1}, 0<x<x_{2}\right)$ for the equations of energy and thermal conductivity, the zero equality conditions of corresponding functions gradients - for the continuity, motion and diffusion equations; the conjugating boundary conditions were prescribed on "fuel vapors - liquid" boundary $\left(y=y_{2}, 0<x<x_{2}\right)$ for the equations of energy and thermal conductivity taking into account the vapor formation, Newmann's boundary conditions taking into account the injection of vapors were prescribed for the continuity, motion and diffusion equations; the conjugating boundary conditions were prescribed on "liquid - vapor-gas mixture" boundary $\left(y=y_{3}, 0<x<x_{2} ; x_{1}<x<\right.$ $x_{2}, r=r_{1}, 0<\varphi<\pi / 2$ ) for the equations of energy and thermal conductivity, Newmann's boundary conditions taking into account the injection of vapors in the form of bubbles were prescribed for the continuity, motion and diffusion equations; the zero equality condition of corresponding functions gradients was accepted on the boundaries $(x=0, x=H, 0<y<L ; y=0, y=L, 0<x<H)$ for all equations.

The vapor bubble-rise velocity $V_{\mathrm{vm}}$ during the boiling depends on the mode of flow and the bubble dimensions $R_{\mathrm{vm}}$. It is known that the bubble-rise detached from the bottom takes place in the turbulent conditions. Since the most part of bubbles in the thin films is formed nearby the heated substrate, then the formula characterizing the turbulent bubble-rise in the shape of sphere in $R_{\mathrm{vm}}$ radius can be used to determine the velocity $V_{\mathrm{vm}}$ in a first approximation:

$$
V_{\mathrm{vm}}=\sqrt{\frac{8 R_{\mathrm{vm}} g_{y}}{3}}
$$

The dimensions of rising bubbles for the films of liquids in thickness less than $10 \mathrm{~mm}$ under the conditions of intense evaporation, as a rule, are variable in the range of $0,05<R_{\mathrm{vm}}<1 \mathrm{~mm}$. Therefore, the average bubble-rise velocity is $0,035<V_{\mathrm{vm}}<0,15 \mathrm{~m} / \mathrm{s}$.

The vapor gap dimensions between the substrate and the liquid film (Fig. 1), the mass rate of evaporation and oxidation were calculated similar to [1-3]. The $[6,7]$ models were used to describe the macroscopic regularities of diffusive and convective mass transfer of fuel vapors in the oxidizing agent area in "flow function - vortex velocity vector" variables.

The numerical simulation algorithms and the result adequacy estimation procedure (based on the conservation checking of being used difference scheme) of theoretical investigations carried out are similar to the ones being used in [1-3]. 


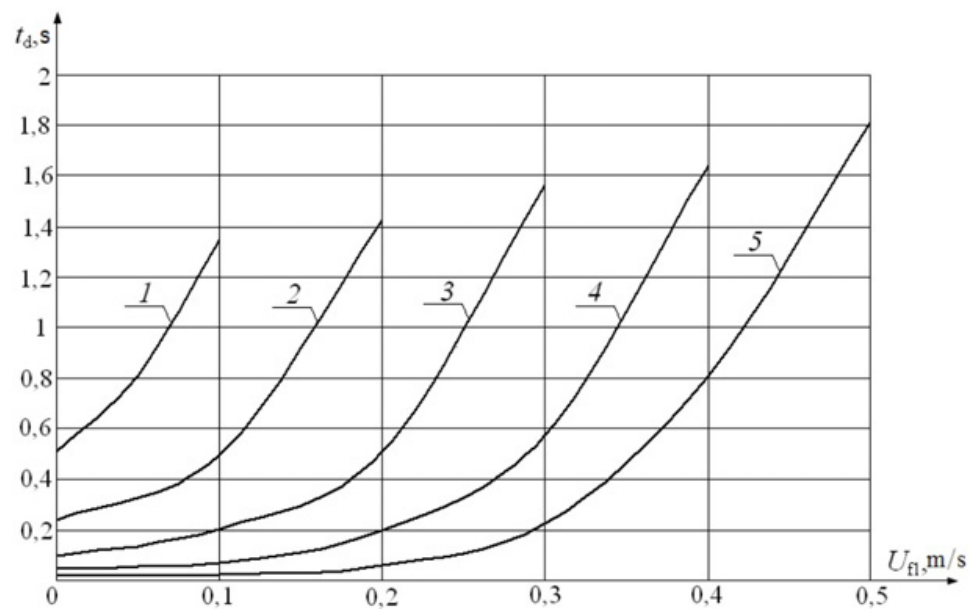

Figure 2. The dependences of ignition delay time on the spilling velocity $U_{\mathrm{ff}}$ at $L_{\mathrm{fl}}=5 \mathrm{~mm}: 1-T_{\mathrm{bm}}=800 \mathrm{~K}$, $2-T_{\mathrm{bm}}=900 \mathrm{~K}, 3-T_{\mathrm{bm}}=1000 \mathrm{~K}, 4-T_{\mathrm{bm}}=1100 \mathrm{~K}, 5-T_{\mathrm{bm}}=1200 \mathrm{~K}$.

\section{Results and discussion}

The numerical simulations were carried out at the following values of parameters [8-10]: liquid and air temperatures are $T_{0}=300 \mathrm{~K}$, metal substrate temperature is $T_{\mathrm{bm}}=600 \div 1300 \mathrm{~K}$; liquid boiling temperature is $T_{\mathrm{bl}}=470 \mathrm{~K}$; heat effect of fuel vapor oxidation reaction in the air is $Q_{\mathrm{o}}=43,8 \mathrm{MJ} / \mathrm{kg}$; heat effect of liquid evaporation is $Q_{\mathrm{e}}=25 \mathrm{~kJ} / \mathrm{kg}$; activation energy of oxidation reaction is $E=$ $193,7 \mathrm{~kJ} / \mathrm{mole}$; pre-exponential factor of oxidation reaction is $k_{0}=7 \cdot 10^{7} \mathrm{~s}^{-1}$; substrate thickness is $L_{\mathrm{bm}}=10 \mathrm{~mm}$; liquid layer thickness is $L_{\mathrm{fl}}=1 \div 10 \mathrm{~mm}$; solution region dimensions are $H=1 \mathrm{~mm}$, $L=50 \mathrm{~mm}$; bubble-rise velocity is $V_{\mathrm{vm}}=0,05 \mathrm{~m} / \mathrm{s}$; liquid spilling velocity is $U_{\mathrm{fl}}=0,1 \div 0,5 \mathrm{~m} / \mathrm{s}$. The thermal and physical characteristics of corresponding matters are presented in [10].

The preanalysis of the ignition process under study shows that the implementation of ignition conditions in the system under consideration (Fig. 1) depends, primarily, on the substrate temperature, the spilling liquid layer thickness and the spilling velocity. Figure 2 shows the dependences of the main integral ignition characteristic (delay time $t_{\mathrm{d}}$ ) on the liquid fuel spilling velocity $U_{\mathrm{ff}}$ for several characteristic substrate temperatures $T_{\mathrm{bm}}$. It was demonstrated that the $t_{\mathrm{d}}=t\left(U_{\mathrm{fl}}\right)$ dependences are drastically exponential. At that, it is significant that the $t_{\mathrm{d}}=t\left(U_{\mathrm{fl}}\right)$ dependences become almost linear (for example, at $T_{\mathrm{bm}}=900 \mathrm{~K}$ ) when decreasing of a substrate temperature. It can be explained by the implementation of ignition conditions at lower temperatures in the reaction zone is determined in a greater degree by the concentration growth of vapor-gas mixture constituents. The dependence of chemical reacting (oxidation) velocity on the concentration of binary (oxidizing agent, fuel) mixture constituents at the numerical simulation was accepted as a linear dependence (first-order reaction was considered).

Also, it is found that the ignition conditions are implemented when increasing of a substrate temperature even with sufficiently large $\left(U_{\mathrm{fl}}=0,5 \mathrm{~m} / \mathrm{s}\right)$ spilling velocities (Fig. 2). It can be explained by the bulk of liquid matter vapors is blown in the oxidizing agent area from the vapor gas area (Fig. 1) into the front of spilling layer of liquid fuel. At that, the numerical analysis showed that the liquid fuel layer "blocks" the output of vapors into the front under the conditions of spilling. Therefore, the concentration of vapors in the gas area is increased sufficiently slowly with the growth of spilling velocity. It leads to the ignition response time increase. The oxidizing agent (air) is warmed up before the beginning of intense chemical reacting with fuel vapors under the conditions of high $\left(T_{\mathrm{bm}}>1100 \mathrm{~K}\right)$ 


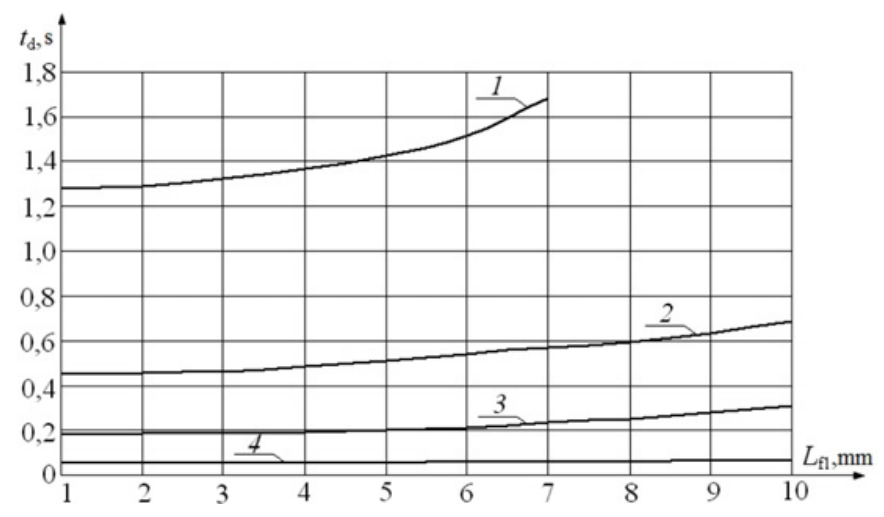

Figure 3. The dependences of ignition delay time on the layer thickness of liquid fuel at $U_{\mathrm{fl}}=0,2 \mathrm{~m} / \mathrm{s}: 1-$ $T_{\mathrm{bm}}=900 \mathrm{~K}, 2-T_{\mathrm{bm}}=1000 \mathrm{~K}, 3-T_{\mathrm{bm}}=1100 \mathrm{~K}, 4-T_{\mathrm{bm}}=1200 \mathrm{~K}$.

substrate temperatures. It is found that the high oxidizing agent temperature and the relatively weak concentration of liquid vapors in the vapor-gas mixture nearby the substrate surface are sufficient to accelerate the reaction of chemical reacting and to implement the ignition conditions (Fig. 2) under such conditions.

Figure 3 shows the ignition delay time dependences on the spilling layer thickness $L_{\mathrm{fl}}$ of liquid fuel for several characteristic substrate temperatures at the relatively low $\left(U_{\mathrm{fl}}=0,2 \mathrm{~m} / \mathrm{s}\right)$ spilling velocity. It is found that the liquid thickness in the system under consideration (Fig. 1) influences slightly on the integral ignition characteristics at high $\left(T_{\mathrm{bm}}>1100 \mathrm{~K}\right)$ substrate temperatures. The $L_{\mathrm{fl}}$ influence on $t_{\mathrm{d}}$ is strengthened when decreasing of the substrate temperature to $900 \mathrm{~K}$. Obtained dependences (Fig. 3) can be explained by the inertial processes of boundary layer warming up of liquid matter and evaporation play an important role at the relatively low substrate temperatures. The ignition conditions are implemented even with warming up (during $t_{\mathrm{d}} \ll 1 \mathrm{~s}$ time) of very thin boundary liquid layer and, consequently, small thickness of vapor gap when increasing of the substrate temperature (Fig. 1). So, for example, Fig. 4 shows the temperature field at the instant of ignition at $T_{\mathrm{bm}}=1200 \mathrm{~K}$. It can be seen that the thickness of vapor gap and boundary layer (heated up to $T_{\mathrm{bl}}$ temperature) of liquid matter does not exceed $0,5 \mathrm{~mm}$ at $L_{\mathrm{fl}}=5 \mathrm{~mm}$ during $t_{\mathrm{d}}=0,058 \mathrm{~s}$. Therefore, $L_{\mathrm{fl}}$ variation in sufficiently wide range $(1 \div 10 \mathrm{~mm})$ does not lead to the significant change of $t_{\mathrm{d}}$.

Figure 4 shows that the zone of basic oxidation reaction is formed into the front of spilling liquid in close proximity to the boundary $\left(x=x_{2} ; y_{1}<y<y_{2}\right)$ of matter vapor injection (Fig. 1). It is found that the ignition zone is slightly displaced from the substrate surface to "liquid - vapor-gas mixture" boundary $\left(y=y_{3}, 0<x<x_{2} ; x_{1}<x<x_{2}, r=r_{1}, 0<\varphi<\pi / 2\right)$ when increasing of spilling velocity $U_{\mathrm{fl}}$. It can be explained by the output of liquid vapors "is blocked" by the impinging liquid layer when increasing of $U_{\mathrm{fl}}$. The vapors are chilled and blown in oxidizing agent area. At the same time, the thickness of the liquid layer warmed up grows. It leads to the concentration growth of vapor bubbles in the spilling liquid. Consequently, the concentration of matter vapors being blown not only in a liquid motion front but from the surface of the fuel is increased. The oxidizing agent is heated up to high temperatures by the heat removal from a substrate. Therefore, the ignition conditions are implemented nearby the boundary of vapor injection from the surface of the fuel.

The analysis of Figs. 2-4 carries inference that the interacting processes between spilling layer of liquid fuel and metal substrate heated are characterized by the short ignition delay times (short response time) at the substrate temperature variation, the layer thickness of spilling liquid and the spilling velocity in sufficiently wide ranges. The selected ranges correspond with many technological 


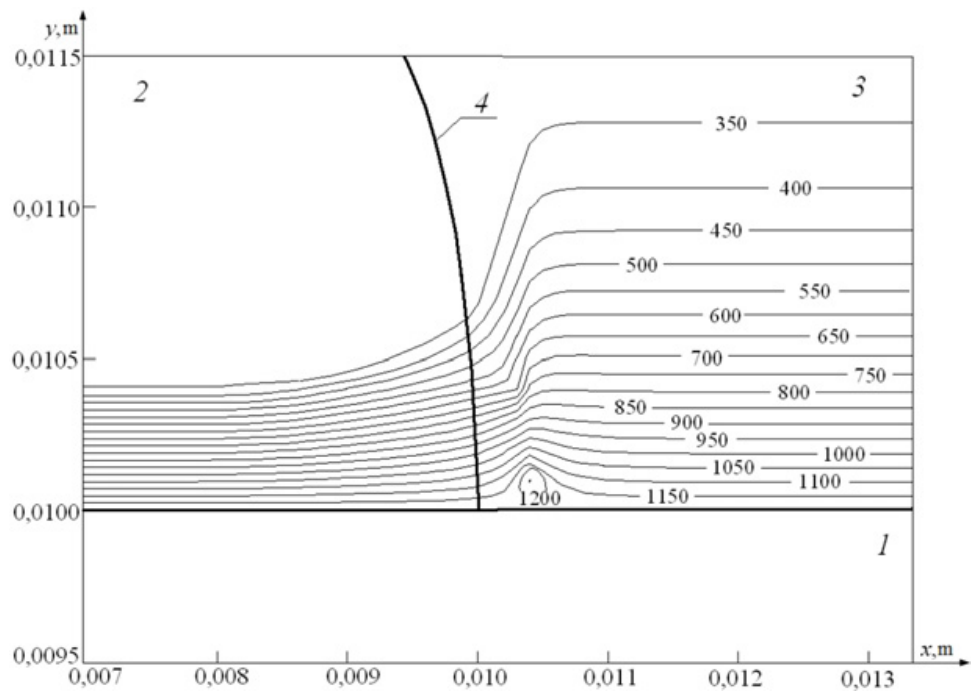

Figure 4. The temperature field at the instant of ignition $\left(t_{\mathrm{d}}=0,058 \mathrm{~s}, T_{\mathrm{bm}}=1200 \mathrm{~K}, U_{\mathrm{fl}}=0,2 \mathrm{~m} / \mathrm{s}, L_{\mathrm{fl}}=5 \mathrm{~mm}\right)$ : 1 - metal substrate, 2 - liquid layer with bubbles of vapors, 3 - oxidizing agent-liquid vapor mixture, 4 - "liquid vapor-gas mixture" boundary.

processes at the places of production and pre-emergency and emergency situations. If it is remembered that the macroscopic ignition regularities of combustible and flammable liquid matters are similar, and the flame propagation velocities over its surface are great, then it is possible to deduce that the spilling of combustible (first of all, fuels) and unappropriated for combustion flammable liquids (varnishes, paints, solvents) over the surfaces heated up to high temperatures poses a high fire danger.

\section{Conclusions}

The dependences of integral characteristics of typical liquid fuel ignition on the metal substrate temperature, the layer thickness of spilling liquid and the spilling velocity were found as a consequence of numerical investigations. It was demonstrated that the ignition is possible even with sufficiently high $(0,5 \mathrm{~m} / \mathrm{s})$ velocities of liquid fuel spilling. It was revealed that the layer thickness of liquid matter insignificantly influences on the integral characteristics of ignition (particularly at high (more than $1000 \mathrm{~K}$ ) substrate temperatures). Also, the conditions $\left(T_{\mathrm{bm}}<600 \mathrm{~K}, U_{\mathrm{fl}}>0,5 \mathrm{~m} / \mathrm{s}, L_{\mathrm{fl}}>5 \mathrm{~mm}\right)$, whereby the impinging liquid layer "blocks" the output of fuel vapors and impedes the ignition, were determined.

The investigation was performed by Russian Science Foundation grant (project 14-39-00003).

\section{References}

[1] G.V. Kuznetsov and P.A. Strizhak, Journ. of Eng. Thermophys. 3, 19 (2009)

[2] D.O. Glushkov, G.V. Kuznetsov, and P.A. Strizhak, Russ. Journ. of Phys. Chem. B 6, 5 (2011)

[3] D.O. Glushkov, G.V. Kuznetsov, and P.A. Strizhak, Math. Probl. in Eng. 2014 (2014)

[4] T.Y. Xiong and M.C. Yuen, Int. Journ. of Heat Mass Transf. 34 (1991) 
[5] V.N. Vilyunov and V.E. Zarko, Ignition of Solids (Elsevier Science Publishers, Amsterdam, 1989)

[6] D.O. Glushkov, G.V. Kuznetsov, and P.A. Strizhak, Adv. in Mech. Eng. 2014 (2014)

[7] O.V. Vysokomornaya, G.V. Kuznetsov, and P.A. Strizhak, Math. Probl. in Eng. 2014 (2014)

[8] N.B. Vargaftik, L.P. Filipov, A.A. Tarzimanov, and E.E. Totskii, Handbook of Thermal Conductivity of Liquids and Gases (CRC Press, Inc., Boca Raton, 1994)

[9] J. House, Principles of Chemical Kinetics (Academic Press, New York, 2007)

[10] V. Patel, Chemical Kinetics (InTech, Rijeka, 2012) 